\title{
Unveiling the nature of the brightest $z>6$ galaxies with ALMA and JWST
}

\section{Rebecca Bowler}

University of Oxford, United Kingdom

\begin{abstract}
The very brightest $z>6$ galaxies are ideal laboratories for studying the physical properties of star-forming objects into the epoch of reionization. Selected from degree-scale, ground-based fields, these rare objects provide a key insight into early dust production and may harbour faint AGN. Targeted follow-up of small samples have unexpectedly shown both Lyman-alpha emission and other rest-frame UV lines (e.g CIV and HeII), suggesting unique starformation conditions (or AGN) at early times. Furthermore, ALMA observations have revealed that $75 \%$ of the star-formation in these galaxies may be obscured. I will talk about HST/ALMA follow-up of bright $z \sim 7$ LBGs in COSMOS and present new results from even brighter samples from $z=6-9$ selected over $\sim 5 \mathrm{deg}^{2}$. The power of both ALMA and JWST, coupled with the intrinsic luminosity of these sources, will provide a unique insight into the formation and evolution of vigorously star-forming galaxies in the first billion years.
\end{abstract}

